\title{
Structure-Activity Relationships of Components from the Roots of Pueraria thunbergiana Having Aldose Reductase Inhibitory and Antioxidative Activity
}

\author{
Chang-Hun Park, Soon Sung Lim, ${ }^{*}$ and Dong-Ung Lee \\ Department of Biotechnology, Dongguk Linversity: Gyeongiu 780-714, Korea. ${ }^{\star}$ E-mail: dulee Gidonggukackr \\ ${ }^{\dagger}$ Silver Biotechnologv Research Center. Hallw Cniversity, Chunchon 200-702, Korea \\ Received October 22, 2006
}

Key Words : Pueraria thumbergiana. Flavonoid. Isoflavonoid. Aldose reductase. Antioxidation

The roots of Pteratia thinbergiana (Leguninosae) have been used in traditional medicine in Korea. Japan and China for the prevention and treatment of common colds because of their antipyretic and analgesic effects. In contrast. the flowers of this plant have mainly been prescribed to treat diabetes mellitus. ${ }^{1}$ In connection with the present study, the roots of this plant were investigated for antioxidative effects, ${ }^{2}$ but their inhibitory activities on aldose reductase have not yet been reported. Aldose reductase (AR), the key enzyme of the polyol pathway, has been demonstrated to play an important role in the etiology of diabetic conlplications: therefore, AR inhibitors would be expected to be effective in preventing cataract formation in diabetes mellitus. ${ }^{3.4}$ It has been established that diabetes is associated with increased oxidative stress. ${ }^{5}$ Therefore. the finding of AR inlibitors that possess antioxidant activity would seem to be desirable, because it has been suggested that increased oxidant production by metal-catalyzed glucose oxidation may be an important mechanism in the activation of $\mathrm{AR}^{6}{ }^{6} \mathrm{In}$ addition. several recent reports demonstrate induction of a large number of genes. including genes related to $A R$, on exposure of cells to oxidative stress. ${ }^{7.8}$

Naturally occurring and synthetic flavonoids have been shown to exhibit antioxidant activity and inhibitory effects on aldose reductase. ${ }^{\text {li-12 }}$ As part of our ongoing search for aldose reductase inhibitors or antioxidants from natural sources. ${ }^{1 / 14}$ we evaluated both activities of the fractions and constituents from the roots of $P$. thmbergiana. Biologically active constituents of the roots of this plant have mainly been examined for isoflavonoids: besides daidzein and daidzin more than 25 isoflavonoids and flavonoids have been isolated. ${ }^{15.16}$ Among the constituents isolated in this study, AR inhibitory activities ${ }^{17}$ and antioxidative activities have already been demonstrated in some compounds, but other analogs and the correlation between both activities in each compound have not yet fully been demonstrated.

Herein. we discuss the structure-activity relationships and the correlation between AR inhibitory activity and antioxidative activity of the components from the roots of $P$. thinbergiana.

The $80 \%$ methanolic extract and fractions of the roots of Pueraria thinbergiana were tested for their inlibitory activities on rat lens aldose reductase (AR) in order to fund the most active fraction. The methylene chloride fraction
Table 1. Inhibitory Activity of the Fractions and Compounds of the Roots of Pueraria thubergiana on Aldose Reductase

\begin{tabular}{lc}
\hline & $\mathrm{IC}_{50}(\mu \mathrm{g} / \mathrm{mL}$ or $\mu \mathrm{M})$ \\
\hline methanol extract & 657 \\
methylene chloride fraction & 1.09 \\
ethylacetate fraction & 1.21 \\
butanol fraction & 8.85 \\
1 & 5.2 \\
$\mathbf{2}$ & 4.5 \\
$\mathbf{3}$ & $>100$ \\
$\mathbf{4}$ & 7.9 \\
$\mathbf{5}$ & 44.7 \\
$\mathbf{6}$ & $>100$ \\
$\mathbf{7}$ & $>100$ \\
$\mathbf{8}$ & 25.1 \\
9 & 15.8 \\
$\mathrm{TMG}^{a}$ & 1.0 \\
\hline
\end{tabular}

TMG (tetramethylene glutaric acid) was tested as a reference compound.

and ethylacetate fraction showed a strong inhibition on $A R$, which showed activities ( $\mathrm{IC}_{5}=1.09$ and $1.21 \mu \mathrm{g} / \mathrm{mL}$. respectively) that were significantly better than those of the other fractions (Table 1). For activity-guided separation. we selected the ethylacetate fraction rather than the methylene chloride fraction. which showed better activity than the ethylacetate fraction. because the ethylacetate fraction revealed much more potent antioxidative activity than did the methylene chloride fraction. From the ethylacetate fraction. we isolated four isoflavonoids, daidzin (3). daidzein (4), puerarin (5). and ononin (6), and identified their structures by comparison of their spectral data with previously reported data. $^{16}$ The effects of the constituents of Pueraria root. including the above compounds 3-6. compound 7 (fomononetin) prepared from compound 3 and the commercial isoflavonoids. genistin (1) and genistein (2), and flavonoids, baicalin (8) and baicalein (9) (Fig. 1). were estimated with rat lens AR using DL-glyceraldehyde as a substrate. As shown in Table I. genistin (1) and genistein (2) possessing a hydroxy group at the C.5 position showed the most potent inhibition of enzyme activity (IC 505.2 and $4.5 \mu \mathrm{M}$, respectively). Daidzein (t) showed much better active than daidzin (3). a glucoside of 4 . and the methoxy-substituted compounds, ononin (6) and formononetin (7). revealed very 


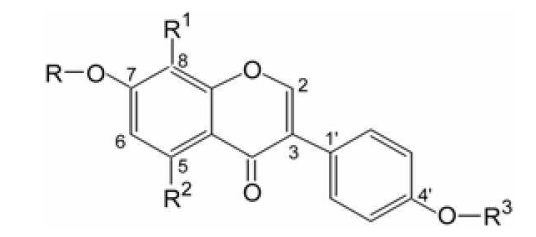

\begin{tabular}{lllll}
\hline Isoflavonoids & $\mathrm{R}$ & $\mathrm{R}^{1}$ & $\mathrm{R}^{2}$ & $\mathrm{R}^{3}$ \\
\hline 1(Genistin) & glu & $\mathrm{H}$ & $\mathrm{OH}$ & $\mathrm{H}$ \\
2(Genistein) & $\mathrm{H}$ & $\mathrm{H}$ & $\mathrm{OH}$ & $\mathrm{H}$ \\
3(Daidzin) & $\mathrm{glu}$ & $\mathrm{H}$ & $\mathrm{H}$ & $\mathrm{H}$ \\
4(Daidzein) & $\mathrm{H}$ & $\mathrm{H}$ & $\mathrm{H}$ & $\mathrm{H}$ \\
5(Puerarin) & $\mathrm{H}$ & $\mathrm{glu}$ & $\mathrm{H}$ & $\mathrm{H}$ \\
6(Ononin) & glu & $\mathrm{H}$ & $\mathrm{H}$ & $\mathrm{CH}_{3}$ \\
7(Formononetin) & $\mathrm{H}$ & $\mathrm{H}$ & $\mathrm{H}$ & $\mathrm{CH}_{3}$ \\
\hline
\end{tabular}

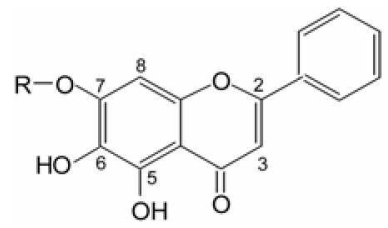

\begin{tabular}{ll}
\hline Flavonoids & $R$ \\
\hline 8 (Baicalin) & glucuronic acid \\
g(Baicalein) & $H$ \\
\hline
\end{tabular}

Figure 1. Chemical Structures of the Tested Compounds.

weak activity. The activity of $C$-glucoside puerarin (5) was also relatively weak. Flavonoids $\mathbf{8}$ and 9 . which included a hy'droxy' group at the C-5 position. like isoflavonoids 1 and 2. exhibited adequate activity. Next antioxidative activities of the fractions and the constituents were investigated. Among the fractions. at a dose of $0.1 \mathrm{mg} / \mathrm{mL}$. the ethylacetate fraction exhibited the most active anti-lipid peroxidation and DPPH radical scavenging potency compared to the control $(10.3 \%$ and $90.4 \%$, respectively). (Table 2). The isolated components (3-6) from the ethylacetate fraction and other isoflavonoids 1.2 and 7 and flavonoids 8 and 9 were evaluated for their inhibitory activities on lipid peroxidation and DPPH radical scavenging activities in vitro and the results are summerized in Table 3 . At a dose of $1 \mu \mathrm{M}$. puerarin (5) and baicalein (9) exhibited anti-lipid peroxidation effects of $31.4 \%$ and $30.5 \%$. respectively: this activity was better than that of $\alpha$-tocopherol, a known antioxidant. at the same concentration. Interestingly, genistin (1) and geni-

Table 2. Inhibitory Activity of the Fractions of the Roots of Pueratia thmberyianta on Lipid Peroxidation and DPPH Radical Scavenging Activity in witro

\begin{tabular}{lrrr}
\hline & \multicolumn{3}{c}{ Concentration $(\mathrm{mng} / \mathrm{mL})$} \\
\cline { 2 - 4 } & \multicolumn{1}{c}{0.1} & \multicolumn{1}{c}{0.25} & \multicolumn{1}{c}{0.5} \\
\hline methanol extract & $<1 / 80.2$ & $2.5 / 88.6$ & $5.2 / 89.3$ \\
methylene chloride fraction & $<1 / 53.1$ & $2.0 / 74.5$ & $5.3 / 78.2$ \\
ethylacetate fraction & $10.3 / 90.4$ & $11.2 / 90.5$ & $15.1 / 92.2$ \\
butanol fraction & $6.5 / 83.7$ & $10.8 / 91.2$ & $12.6 / 93.3$ \\
\hline
\end{tabular}

"Values indicate the $\%$ inhibition on lipid peroxidation: DPPH radical.
Table 3. Antioxidative Activity of Compounds 1-9

\begin{tabular}{|c|c|c|c|c|}
\hline \multirow{2}{*}{ Compound } & \multicolumn{2}{|c|}{$\mathrm{LPO}^{\prime}(\mu \mathrm{M})$} & \multicolumn{2}{|c|}{$\mathrm{DPPH}^{t 1}(\mu \mathrm{M})$} \\
\hline & 2 & l & 2 & 1 \\
\hline 1 & 0.7 & 0.7 & 9.0 & 7.9 \\
\hline 2 & 4.5 & $<0.1$ & 16.4 & 2.6 \\
\hline 3 & 10.6 & 8.2 & 9.0 & 3.7 \\
\hline 4 & 20.8 & 16.2 & 1.5 & 0.7 \\
\hline 5 & 41.9 & 31.4 & 18.4 & 8.1 \\
\hline 6 & 10.4 & 3.1 & 0.8 & 0.6 \\
\hline 7 & 16.4 & 11.9 & 0.4 & 0.2 \\
\hline 8 & 20.3 & 18.8 & 97.0 & 97.0 \\
\hline 9 & 38.9 & 30.5 & 48.2 & 26.1 \\
\hline$\alpha$-tocopherol & 33.1 & 25.2 & 92.6 & 89.0 \\
\hline
\end{tabular}

Anti-lipid peroxidation activity (?.o inhibition in mouse liver homogenate). ${ }^{b}$ Chemically stable DPPH radical scavenging activity (do inhibition?

stein (2). which were most active in the AR activity assay. showed the weakest activity on lipid peroxidation: in contrast. their DPPH radical scavenging activities were remarkable. The anti-lipid peroxidation effect and DPPH radical scavenging activity of the tested compounds displayed no general trend. The antioxidative effects of the ethylacetate fraction may result from the activities of puerarin (5). a $C$ glucoside. Baicalin (8) potently surpressed DPPH radical $(97.0 \%$ inhibition at $1 \mu \mathrm{M})$. However, its anti-lipid peroxidation activity was much weaker than that of 5 . The radical scavenging activity of the flavonoids $(8,9)$ was much better than that of the isoflavonoids (1-7).

Compounds used in this study can be classified into isoflavonoid glucosides $(\mathbf{1}, \mathbf{3}, \mathbf{5}, \mathbf{6})$ and their aglycones (2.4. 7). and flavonoid glucoside (8) and its aglycone (9). The structure-activity relationship studies focused on the effects of substituents on the aromatic ring in the structure. First. an AR activity assay of compounds 1-9 indicated that. without exception. each aglycone inhibited more than its corresponding glucoside. For example. the most potent genistein (2) was more active than its glucoside genistin (1). Moreover. daidzein (4) showed better activity than its $O$-glucoside daidzin (3) and C-glucoside puerarin (5). These results are in good agreement with those reported for compounds 1-4. ${ }^{18}$ Substitution of the methosy group at C.4' (6. 7) strongly reduced the enzyme activity. Next, in the anti-lipid peroxidation assay. aglycones $(\mathbf{2}, 4.7)$ were active than the corresponding glucosides $(\mathbf{1}, \mathbf{3}, \mathbf{6})$. However, their DPPH radical scavenging activities exhibited the opposite tendency. Comparison of the AR inhibitory activity with the antioxidative activity of tested compounds unexpectedly showed that both activities in each compound were not proportional to each other. For example. compounds $\mathbf{1}$ and $\mathbf{2}$ exerted outstanding AR inlibitory activity but revealed very weak anti-lipid peroxidation activity. Compound $\mathbf{5}$, which had relatively weak AR inhibitory activity. showed potent antioxidative activity. As previously noted diabetes is associated with increased oxidative stress. so AR inhibitors possessing antioxidant activity would seem to be desirable compounds for 
use in the treatment of diabetes mellitus. ${ }^{5.6}$ Considering the close association between AR inhibitor and antioxidant. isoflavonoid 4 and flavonoid 9 would be acceptable compounds for which further trials including in vivo testing should be conducted

\section{Experimental Section}

Melting points were measured on an Electrothermal IA9100 apparatus (Reallabware Co. Watford Herts. UK) and are uncorrected. NMR spectra were recorded on a Varian GEMINI-200 (Varian Inc. Palo Alto. CA. USA) spectrometer using $\mathrm{CDCl}_{3}$ as a solvent. All chemical reagents for the activity test and standard compounds baicalein. baicalin. genistein, and genistin were purchased from Sigma Chemical Co. (St. Louis. MO. USA).

Extraction and Isolation. The powdered roots $(600 \mathrm{~g})$ of commercially available Pteraria thmbergiana were extracted with $80 \%$ methanol $(2 \times 2$ L) under reflux. The total extracts $(168 \mathrm{~g})$ were fractionated with $200 \mathrm{~mL}$ of each of methylene chloride. ethylacetate and $n$-butanol to yield 12.6 g. $25.7 \mathrm{~g}$ and $33.8 \mathrm{~g}$. respectively. The active ethylacetate fraction was chromatographed on a silica gel column $(5-40$ $\mu \mathrm{m} .5 \times 70 \mathrm{~cm})($ Merck \& Co.. Inc., Whitehouse Station. NJ. USA) using $\mathrm{CH}_{2} \mathrm{Cl}_{2}=$ methanol (gradient $5: 1$ to $1: 1$ ) as a solvent sy'stem to furnish daidzin (3) daidzein (4). puerarin (5) and ononin (6). for which structures were confirmed by comparison with the known spectral data. ${ }^{16}$

HPLC Analysis. The isolated components were analyzed by reversed-phase ODS-HPLC using a Shimadzu LC-10AD system (Kyoto. Japan): Inertsil ODS (4.6 mm i.d $\times 150 \mathrm{~mm}$ ) column: $0.05 \mathrm{M} \mathrm{Na}_{2} \mathrm{HPO}_{4}$-Acetonitrile 85:15: UV detection $254 \mathrm{~nm}$ : flow rate $0.7 \mathrm{~mL} / \mathrm{min}$ : retention time (min): 3 (12.25). 4 (9.16). 5 (3.10). 6(11.42).

Synthesis of 4'-Methoxydaidzein (7). Daidzin (3.50 mg) was methylated with diazomethane prepared by Diazald $(N$ methyl- $N$-nitroso- $p$-toluenesulfonamide) to give 4 '-methoxydaidzin. which was hydrolyzed with dilute sulfuric acid. giving 4'-methoxydaidzein (formononetin) $(7.8 \mathrm{mg}$ ). which was identified by comparison with reported data. ${ }^{19}$

Preparation of Enzyme Source. Crude rat lens aldose reductase was prepared as follows: rat lenses were removed from Sprague-Dawley rats weighing 250-280 g and frozen until use. The supernatant fraction of the rat lens homogenate was prepared according to Hayman \& Kinoshita $\mathrm{a}^{\text {ib }}$ and then partially purified according to Inagaki et $a l^{\hat{2}}$ Partially purified enzyme with a specific activity of $6.5 \mathrm{mU} /$ Ing was routinely used to test enzyme inhibition. The partially purified material was separated into $1.0 \mathrm{~mL}$ aliquots and stored at $40^{\circ} \mathrm{C}$

Measurement of Aldose Reductase Activity. Rat lens aldose reductase activity was assayed spectrophotometrically by measuring the decrease in absorption of NADPH at $340 \mathrm{~mm}$ over a $4 \mathrm{~min}$ period with DL-glyceraldelyde as a substrate. ${ }^{\Upsilon 2}$ Each $1.0 \mathrm{~mL}$ cuvette contained equal units of enzyme. $0.10 \mathrm{M}$ sodium phosphate buffer $(\mathrm{pH} 6.2), 0.3 \mathrm{mM}$
NADPH with or without $10 \mathrm{mM}$ substrate and inhibitor. The concentration of inhibitors giving $50 \%$ inhibition of enzyme activity $\left(\mathrm{IC}_{50}\right)$ was calculated from the least-squares regression line of the logarithmic concentrations plotted against the remaining activity:

Lipid Peroxidation Assay. According to the method of Ohkawa et al. ${ }^{23}$ tissue homogenates, $8.1 \%$ sodium dodecyl sulfate. $20 \%$ acetate buffer $(\mathrm{pH} 3.5)$ and $0.8 \%$ 2-thiobarbituric acid were incubated for $1 \mathrm{~h}$ at $95^{\circ} \mathrm{C}$. and then cooled to room temperature. The thiobarbituric acid reactive substance (pink color) in the reactant was transferred to a mixture of $n$-butanol:pyridine (15:1) and its absorbance was measured at $532 \mathrm{~nm}$ as the degree of lipid peroxidation.

DPPH Radical Scavenging Activity Assay. According to the method of Blois. ${ }^{2+}$ each of the sample with various concentrations in $\mathrm{MeOH}$ was mixed with $1.5 \times 10^{-4} \mathrm{M} \mathrm{DPPH}$ in $\mathrm{MeOH}$. After standing for $30 \mathrm{~min}$. at RT. the absorbance was measured at $520 \mathrm{~nm}$ and compared with that of the control

\section{References}

1. Kimn. T. T. Konean Resource Plantw: Seoul National University Press: Seoul. 1996; Vol. 2, p. 232

2. Nam. K. A.: Lee, S. K. Natmal Product Sciences 1999, 5. 165 .

3. Beyer-Mears. A.; Cruz, E. Diabetes 1985. 34. 15.

4. Handelsman. D. J.: Turtle. J. R. Diabetes $1981,30.459$.

5. Cerielo. A.: Motz. E.: Caqarape. A.: Lizzio. S.: Russo. A.: Quatraro. A.: Giugliano. D. J. Diab. Compl 1997. H. 250.

6. Ou. P.: Nourooz-Zadeh. T.: Tritschler. H.: Wolff. S. Free Rad. Res. 1996. 25,337

7. Carper. D. A.: Sun. J. K.: Iwata, T.: Jr. Zigler, J. D.; Ibaraki. N.; Lin, L. R.: Reddy. V. Investigative Ophthalnology and Iistal Science 1999. 40.400 .

8. Spycher. S. E.: Tabataba-Vakili. S.: O'Donnell. V. B.: Palomba. L.: AzZi. A. FASEB J. 1997. 11. 181

9. Pietta. P. G. J. Fat. Prod. 2000. 63. 1035.

10. Iwata. S.; Nagata. N.; Omae. A.; Yamaguchi, S.: Okata. Y.: Shibata, S.: Oluvama, T. Biol. Pham Bull. 1999, 22,323.

11. Shin. K. H.: Kang. S. S.: Kim. H. I.: Shin. S. W. Phronedicine 1994. 1. 145.

12. Shit1. K. H.: Kan1. S. S.: Seo. E. A.: Shit1. S. W. Awh. Pharnt. Res $1995.18,65$.

13. Lee. D. U.: Shin. U. S.: Keun. H. Planta Med 1996. $62,485$.

14. Lee. D. W. Kim, C. H.: Lee. D. U. Biol. Pham. Bull. 2001. 24. 1451 .

15. Shibata. S.: Murakami. T.: Nishikawa. Y. Jakngaku Zashi 1959. 79.757

16. Hosny. M.: Rosazza. J. P. N. J. Nat Prod 1999,62.853

17. Shimizu. M; Ito. T.: Terashima. S.: Havashi. T: Arisawa. M.; Morita. N:- Kurokawa. S: Ito, K: Hashimoto, Y. Plyrochem. 1984. 23. 1885 .

18. Matsuda. H.: Morikawa. T.: Toguchida. I.: Yoshikawa. M. Chent. Pharn. Bull. 2002. 50.788

19. Murthy. M. S. R.: Venkata Rao. E.: Ward, R. S. Magn. Reson. Chem 1986, 24, 225 .

20. Hayman. S.: Kinoshita. J. H. J. Biol. Chem. $1965,240.877$.

21. Inagaki. K.: Miwa. L.: Okuda. I. Arch. Biochem. Biophns. 1982. 216.337.

22. Sato. S.: Kador. P. F. Biochen. Phomacol 1990. 40. 1033.

23. Ohkawa. $\mathrm{H}$; Ohishi, N; Yaki. K. Anal. Biochem. 1979, 95, 351

24. Blois. M. S. Kante 1958, 26, 1199 